\title{
Brian E. Penprase, The Power of the Stars (Second Edition)
}

\author{
Cham, Switzerland: Springer (2017). English, 350 pp., with colour
} illustrations. e-Book, ISBN: 978-3-319-52597-6. £31.99. Hardcover, ISBN: 978-3-319-52595-2. £39.99.

\section{Liz Henty \\ University of Wales Trinity Saint David lizhenty2@gmail.com}

This book's title, The Power of the Stars, is a slight misnomer, as its content encompasses all the celestial objects in the sky including the Sun, the Moon and the planets as well as the eponymous stars of both hemispheres. Brian Penprase's first edition in 2010 was more aptly titled The Power of the Stars: How Celestial Observations Have Shaped Civilization, because the volume is really a beginner's guide to cultural astronomy. The reason given for this update is that new tools have been developed both in astronomy and archaeology which have led to greater understanding of the importance of the sky. The clues are often "subtle and ambiguous" (p. 2) and archaeoastronomy is tasked with reconstructing ancient cosmologies using a range of different methodologies. As Penprase points out: "The night sky calls to us and over the years humans responded with their star tales, their monuments" (p. 2). Within the book's many pages are contained numerous and various examples of this very human response to the sky, not just across millennia but also from around the world.

The book is neatly organised into ten chapters, beginning with Chapter 1, "Our Experience of the Sky and Star Knowledge", with the penultimate Chapter 9 before the "Concluding Thoughts" being "The Development of Modern Cosmology", which takes us to present-day astrophysics. This is not necessarily a book to be read through from cover to cover, but rather to be dipped in and out of, and the clear listing of the subsections for each chapter makes it easy to hone in on a particular area of interest. Additionally, at the end of each chapter there is a bibliography of the main references which can be referred to for corroboration and further study if desired. This is a useful device because details of further reading material are made readily accessible, rather than getting lost in a massive bibliography at the end. However, there is a slight anomaly with the search facility for the online version used for this review: if you enter a search term the page number given is not always right, although if you click on the link it does take you to the correct but 
different page. The volume is worth buying for the images alone, which are stunning throughout; probably the best collection of this kind to be found all in one place.

Chapter 1 draws attention to the sheer variety of names given to the Sun (p. 21), which demonstrates two things: firstly, that the Sun has immense cultural importance; and secondly, that it can be appropriated to mean whatever works for a particular culture. For example it is "Odin's Eye" in Norse mythology or a creator god for the Zuni Native American Pueblo peoples. Often astrology is omitted from cultural astronomy books but here the planets are accompanied by their astrological symbols, another recognition of the way the sky can be used to give meaning to human existence. Perhaps this is where the power of the stars comes in, because in these examples the Sun, Moon and planets can be viewed as omnipresent, in many cases to be worshipped, and not least, they can be seen as harbingers of earthly events. In this way Chapter 1 explains the "Human Experience of the Sky", to quote the first subheading of the chapter.

Chapter 2 concentrates on the night sky, and takes a tour round the constellations of the northern and southern hemispheres with sections on circumpolar stars and the equatorial skies of Hawaii and Polynesia, for example. There is a description of the firstmagnitude stars with a summary of their associated sky lore, plus how they were understood differently around the world. As with the Sun, many stars have local and meaningful names, such as the indigenous Hawaiian name for Sirius, "Hokuho'okelewa'a", meaning "star to guide canoe" (p. 68). Other cultures, such as the Navajo, created constellations that had little in common with our western ones, and in their culture the circumpolar stars were seen as a couple: "First Man and First Woman, revolving round a camp fire" (p. 70).

The current trend for specialisation tends to blind us to the sheer variety of cultural worldviews and cosmologies, so it is good to see so many examples juxtaposed and interesting to learn how many of them contain the same common threads. The creation stories from around the world detailed in Chapter 3 are similarly diverse and imaginative. They feature tales of a "primordial creator" (p. 88), a "cosmic egg" (p. 89), and "The Dreamtime" (p. 102) of the Australian aboriginals, or creations born out of natural disasters such as floods (p. 103). These tend to be more aesthetically appealing than stories of the "Big Bang". Different cosmologies are crafted from these creation myths, and Chapter 4 looks at historical models of the universe, some of which - for example those of the ancient Greek and Chinese - were remarkably close to our own, while others - such as the Mayan and the Babylonian 13 levels of the underworld - seem fantastic (p. 114). The tour includes Norse, Chinese, Hindu, Buddhist, Mayan, Native North American and African cosmologies. As with the creation stories these topics are addressed from the point of view of an informative narrative, rather than through comparison, contrast and discussion.

The importance of the need to regulate and measure time for economic and religious activities is addressed in Chapter 5, which examines calendars. It was astronomical record keeping that enabled the development of calendars from the earliest times ( $p$. 149); the oldest discovered lunar calendar, found by Alexander Marshack, consists of notches on a fragment of bone around 33,000 years old. Solar calendars became useful 
ways of coordinating the times for planting or harvesting of crops. A more sophisticated problem was matching the solar and lunar calendars, but this was achieved by the Babylonians with the device of an "intercalary" month when necessary. The Egyptians solved the problem by adding an extra month dependent on the heliacal rising of Sirius (p. 153) whereas the Hawaiians referenced the position of the Pleiades to divide the year into two equal sections, summer and winter (p.157). Both the Hebrew and Islamic calendars start their months from the first sighting of the crescent Moon, while others reflect particular cosmological and mythological traditions; examples here include calendars that divide according to regular terrestrial events such as the flooding of the Nile in Egypt or the commencement of the dry summer winds for the Chumash peoples. The Roman Julian calendar is apparently still used in astronomy research where "the complexities of orbital periods require the use of a uniform count of days" (p. 165) - this is the calendar that was amended in Catholic Europe in 1582 into the Gregorian calendar used around much of the world today.

Chapter 6 concentrates on timekeeping and clocks. While this might seem out of the scope of the rest of the book, these elements are important, Penprase argues, because the development in the modern world of technology, science and material culture was "inseparable" from the development of the science of time (p. 185). The online version used for this review repeats two of the opening sentences, which is clearly an editorial error, but this should not distract from the accounts of shadow clocks, sundials, navigations devices and so on, right up to the modern-day atomic clock. The history of calendars started in Chapter 5 continues in Chapter 6 with details of the French Revolutionary calendar from two centuries ago and ends with the World Calendar proposed by Elisabeth Achelis in 1930 - a reform that was never implemented.

The term "celestial architecture" (p. 207) is used in Chapter 7 to describe the variety of monuments that are aligned to events in the sky before the narrative goes on to detail some of the best known, such as Stonehenge, Newgrange and the variety of Native American alignments and monuments which include pueblo kivas, the Cahokia mounds and the Chaco Canyon alignments. The Pyramids of Giza, the Kaaba in Mecca, the alignments of ancient Indian Temples, Mayan sites such as Teotihuacan, the temple complex at Angkor Wat in Cambodia are all described. That celestial alignments feature in so many different ancient cultures is well known, but Penprase also includes the Pantheon in Rome, the Parthenon of Athens and the medieval cathedral at Cologne as being cosmologically ordered. In this sense the chapter, like the others, is encyclopaedia-like, briefly summarising some of the known literature. However, it is disappointing that the editors did not pick up that "Callenish" (p. 210), spelled elsewhere in the book as "Callinash" (p. 250) should now be properly referred to as "Calanais" or that the "Sommerville" referred to should have been Vice-Admiral Boyle Somerville, with one " $m$ ". The references for this chapter are patchy - I would certainly like to read the 1973 paper by Alexander Thom referred in the text which apparently mentioned triangles with "numerical ratios of 1:3:10" (p. 250), but none of Thom's publications appear in the list of references for this chapter. Further, although Thom did publish two papers in 1973, one on the Ring of Brodgar in Orkney and the other on the cromlechs at Kerlescan in the Carnac area, it is 
only the latter that mentions Pythagorean triangles, and only in the ratios of $23,25,34$; $38,41,56$ and 28, 53, 60 (Thom 1973, 172). Apologies for the pedantry, but this began to sow seeds of doubt in my mind about the volume's overall accuracy.

Archaeoastronomy in present-day culture is often overlooked in archaeoastronomy compendia, so Chapter 8 , which gives an overview of how it is practised in modern civilisation today, is a welcome addition. Analysing skyscrapers seems a good place to start. The Makkah Royal Clock Tower close to the Kaaba at Mecca includes two moon-viewing galleries, while the Taipei 101 tower in Taiwan is aligned to the cardinal directions (p. 256). The Sears Tower in Chicago is also included as it faces the equinox sunrise, although this may be a pragmatic solution because the entire city is locked into a cardinally aligned grid (p. 257). Manhattanhenge allows winter solstice sunrise to be viewed from many of Manhattan's streets. Penprase does not explore whether these examples are "intentional" or "fortuitous", and this is a subject which is worthy of more research. In his exploration of the present day "skyspace", Penprase is to be commended for his inclusion of James Turrell's art installations which have explored the "interfaces between observer and light, earth and sky" (p. 261). The "Sun Marker" installation (Richman et al. 2016), featured in JSA 2.2, would have fitted perfectly into this chapter. From examining art as skyspace he continues by looking at modern replicas of Stonehenge and juxtaposes these with "Carhenge", "an automotive tribute to Stonehenge" (p. 263), before travelling on to some impressive sundials. As part of his panorama of modern cosmology he details a brief history of Western, Indian and Chinese astrology before asking the question of why people believe in astrology in the twenty-first century - yet far from answering this, he simply lists the well-known arguments against its validity.

Before Penprase draws the book to its conclusion, Chapter 9 delves into the history of astronomy/cosmology and notes the importance of key figures such as Copernicus, Kepler, Galileo and Newton before exploring the astrophysical "worlds" of Herschel and Hubble. This is the scientific view of the universe, which has little to do with the different cosmologies through time and culture that he has taken great pains to detail in the preceding chapters, as it goes way beyond the solar system and the visible stars into the furthest reaches of space. Whereas earlier cultures saw with their own eyes, here he is dealing with what lies through the telescope and beyond. This overview of current knowledge fits with Penprase's aim to examine "the power of the stars" as he concludes that in each culture "the response to the stars mirrored the environment, history, and belief system of the people and provided a means to bind them together" (p. 333). But though that might well be true it is difficult to accept his view that modern cosmology has given us answers to the questions sought by the ancients (p. 337). It is doubtful indeed if the "ancients" wondered how old the universe was or where it was going.

This brief overview of the chapters merely picks out highlights from a wealth of detail gleaned from cultures throughout the world, so if you are wondering about the Mayan long-count calendar, the Dogon cosmology or the Antikythera mechanism then you need to read the book itself - as you will find these and much more within its pages. 


\section{References}

Richman, A. M., V. D. Chamberlain and J. Pachak, 2016. "'Sun Marker': A Laboratory for Experiential Cultural Astronomy". Journal of Skyscape Archaeology 2 (2): 217-224. https://doi.org/10.1558/jsa.30373

Thom, A. and A. S. Thom, 1973. "The Kerlescan Cromlechs". Journal for the History of Astronomy 4 (3): 168-173. https://doi.org/10.1177/002182867300400303 